\title{
Acute traumatic aortic rupture: A comparison of surgical and stent-graft repair
}

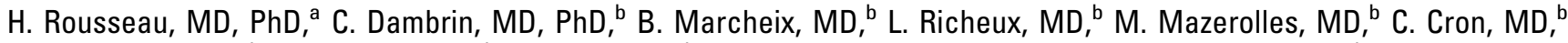 \\ A. Watkinson, MD, ${ }^{b}$ A. Mugniot, MD, ${ }^{b}$ P. Soula, MD, ${ }^{b}$ V. Chabbert, MD, ${ }^{a}$ G. Canevet, MD, ${ }^{a}$ D. Roux, MD, \\ P. Massabuau, MD, ${ }^{\mathrm{c}}$ G. Meites, MD, ${ }^{\mathrm{a}} \mathrm{T}$. Tran Van, MD, and P. Otal, MD, PhD ${ }^{\mathrm{a}}$
}

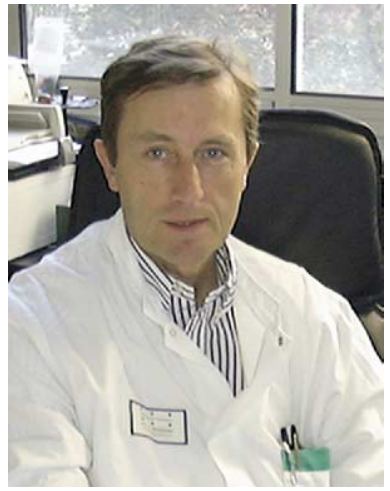

Dr Rousseau

Objective: The study's objective was to comparatively evaluate surgery and stentgraft repair of acute or subacute traumatic aortic rupture.

Methods: A total of 76 patients (14-76 years old; mean, 37 years; male/female ratio, 63/11) with a traumatic aortic injury were admitted to our hospital between 1981 and 2003. Six patients died within 1 to 9 days of another associated severe traumatic lesion. The 70 remaining patients were divided according to the type of rupture repair. In group 1, 35 patients were treated surgically: 28 with immediate repair and 7 with delayed repair (average time interval 66 days, 5-257 days). In group 2, 29 patients were treated with stent grafting of the aortic isthmus. In group 3, 6 patients with minor aortic lesions were treated medically with a close follow-up.

Results: In the 28 patients treated surgically in the emergency department, the mortality and paraplegia rates were $21 \%$ and $7 \%$, respectively. No death or paraplegia was observed in the group with delayed surgical repair. With stent grafting, complete exclusion of the pseudoaneurysmal sac was observed in all patients. Except for 1 iliac rupture treated during the same procedure, there was no major morbidity or mortality during the mean follow-up of 46 months (13-90 months). No major complication was observed in group 3 .

Conclusions: In stable rupture of the aorta, initial conservative treatment is safe and allows management of the major associated lesions. Stent grafting of the aortic isthmus is a valuable therapeutic alternative to surgical repair, especially in patients considered high risk for conventional thoracotomy.

From the Departments of Radiology, ${ }^{\text {a }}$ Cardiovascular Surgery, ${ }^{\mathrm{b}}$ and Cardiology, University Hospital Rangueil, Toulouse, France.

Received for publication Oct 26, 2004; revisions received Dec 13, 2004; accepted for publication Dec 20, 2004

Address for reprints: H. Rousseau, MD, University Hospital Rangueil, 01 av J Poulhes, 31403 Toulouse, France (E-mail: rousseau.h@ chu-toulouse.fr).

J Thorac Cardiovasc Surg 2005;129:1050-5 $0022-5223 / \$ 30.00$

Copyright (C) 2005 by The American Association for Thoracic Surgery

doi:10.1016/j.jtcvs.2004.12.023
$\mathrm{T}$ he traditional management of acute traumatic rupture of the thoracic aorta has been repair of the injury as soon as possible. The recommendation for this treatment is primarily based on the premise that as many as $90 \%$ of patients will die within the first 24 hours after their injury. ${ }^{1,2}$ Without appropriate treatment, $30 \%$ of survivors who reach the hospital die within the first 6 hours. ${ }^{3}$ Although techniques in aortic surgery have improved over the years, morbidity and mortality rates still remain high. ${ }^{4}$ This high death rate is often the result of the other associated injuries that may be present in the patient who has experienced major trauma. Delayed repair of the acute aortic tear, allowing, in select cases, the patient to recover from other major injuries, has been reported in the literature with increasing frequency. ${ }^{5-9}$

More recently, with the introduction of endoluminal aortic stent grafting, a new strategy was introduced for treatment of chronic aortic diseases, but little is known about endovascular repair performed in an acute or subacute setting. ${ }^{10,11}$ The goal of endovascular stenting is to provide a durable exclusion of the lesion while minimizing the morbidity and mortality of the open procedure. The advantages include the avoidance of thoracotomy, significant heparinization, and possible lower risk of paraplegia. 

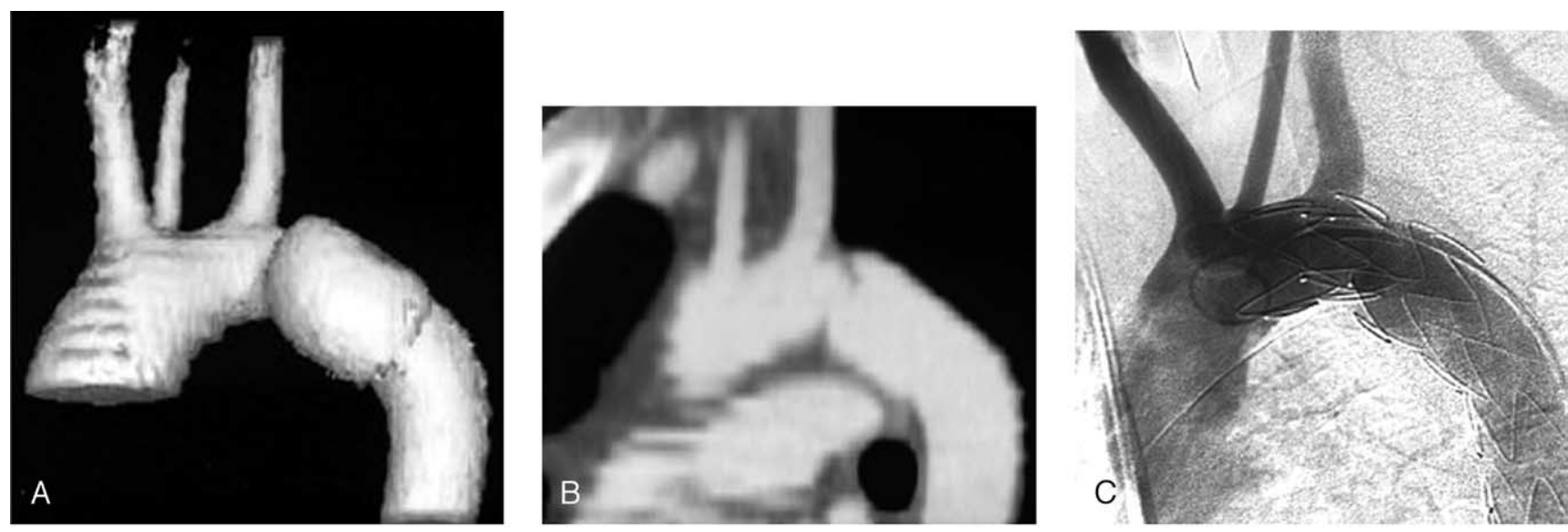

Figure 1. A and B, Spiral CT shows a typical aortic isthmus injury before stent graft. C, Angiographic control immediately after the intervention shows the proximal extremity of the device soon after the left carotid artery ostium, with a complete exclusion of the pseudoaneurysm. There is a slight indentation of the Dacron at the site of the neck.

The purpose of the present study was to compare the implantation of stent grafts with open surgery, in the acute or subacute phase. A new strategy will be proposed.

\section{Methods}

The records of 76 consecutive patients with rupture of the thoracic aorta (Figure 1, $A$ and $B$ ) from blunt trauma after a motorbike or car accident who were treated between 1981 and 2003 were retrospectively reviewed. Six patients died within 24 hours and 9 days of another associated severe traumatic lesion (3 neurologic complications, 2 abdominal injuries, and 1 sepsis infection). The 70 remaining patients were divided into 3 groups according to the type of rupture repair. Patients in group 1 underwent surgical repair of the rupture. Patients in group 2 were treated with stent

TABLE 1. Comparison of Injury Severity Score and complication rates of each group

\begin{tabular}{lccc}
\hline Type of treatment & $\begin{array}{c}\text { ISS } \\
\text { Mean } \pm \text { SE }\end{array}$ & Mortality & Paraplegia \\
\hline Abstention N =6 & $37.8 \pm 4.0$ & 6 & 0 \\
Surgery N = 35 & $33.0 \pm 1.9$ & 6 & 3 \\
Stent grafts N = 29 & $35.0 \pm 2.1$ & 0 & 0 \\
Medical therapy N =6 & $39.6 \pm 4.1$ & 0 & 0 \\
Total N =76 & $35.5 \pm 1.5$ & 12 & 3 \\
\hline
\end{tabular}

ISS, Injury Severity Score. An anatomic scoring system was used. ${ }^{12}$ The body was divided in 6 regions, and a score was assigned for each injury. Only the highest score in each body region was used. The 3 most severely injured body regions had their score squared and added together to produce the ISS (from $0-75$ ). The mortality rate differences were considered statistically significant between Surgery and Stent grafts $(P<.05)$ and Abstention and Stent grafts $(P<.001)$. There were no statistically significant differences for ISS scores and paraplegia rate between the different groups. grafts. Patients in group 3 were treated with medical therapy alone. Results were expressed as mean $\pm \mathrm{SE}$, unless otherwise specified.

The maximum size of the pseudoaneurysmal sac ranged from 26 to $70 \mathrm{~mm}$ (mean, $42.2 \mathrm{~mm}$ ). All patients had at least 2 additional severe lesions, including lung contusion and/or serial rib fractures with reduced respiratory function, cerebral lesions with neurologic deficits, abdominal visceral lacerations, and extremity or pelvic fractures. An anatomic scoring system, the Injury Severity Score [ISS], from 0 to 75 was used (Table 1). ${ }^{12}$

\section{Therapeutic Strategy}

On their arrival in the intensive care unit, patients were immediately submitted to intensive resuscitation with the establishment of electrocardiography and continuous monitoring of arterial and central venous pressure, renal function, and other hemodynamic and clinical measurements. Medical therapy consisted of $\beta$-blocking agents (metoprolol) and arterial vasodilators (nitroprusside and calciumblocking drugs). Intravenous fluid replacement with a number of different compounds was given to maintain the systolic blood pressure at less than $120 \mathrm{~mm} \mathrm{Hg}$. Once the patient was hemodynamically stable, antihypertensive therapy was given orally. The delay and choice of treatment were dictated by the general condition of the patient, surgical risk factors, and type of aortic trauma.

\section{Surgical Group 1}

In our series, 35 patients underwent surgical repair of the aortic rupture.

In group 1a, 28 patients underwent emergency surgery within 48 hours after the trauma. An aortic prosthesis was positioned about the isthmus in 19 patients, and direct suturing of the isthmus was performed in 9 patients. In 17 patients, a partial bypass through the femoral vein and pulmonary artery to the femoral artery was used. In 11 patients, surgery was performed without cardiopulmonary support by using a simple clamping technique. 

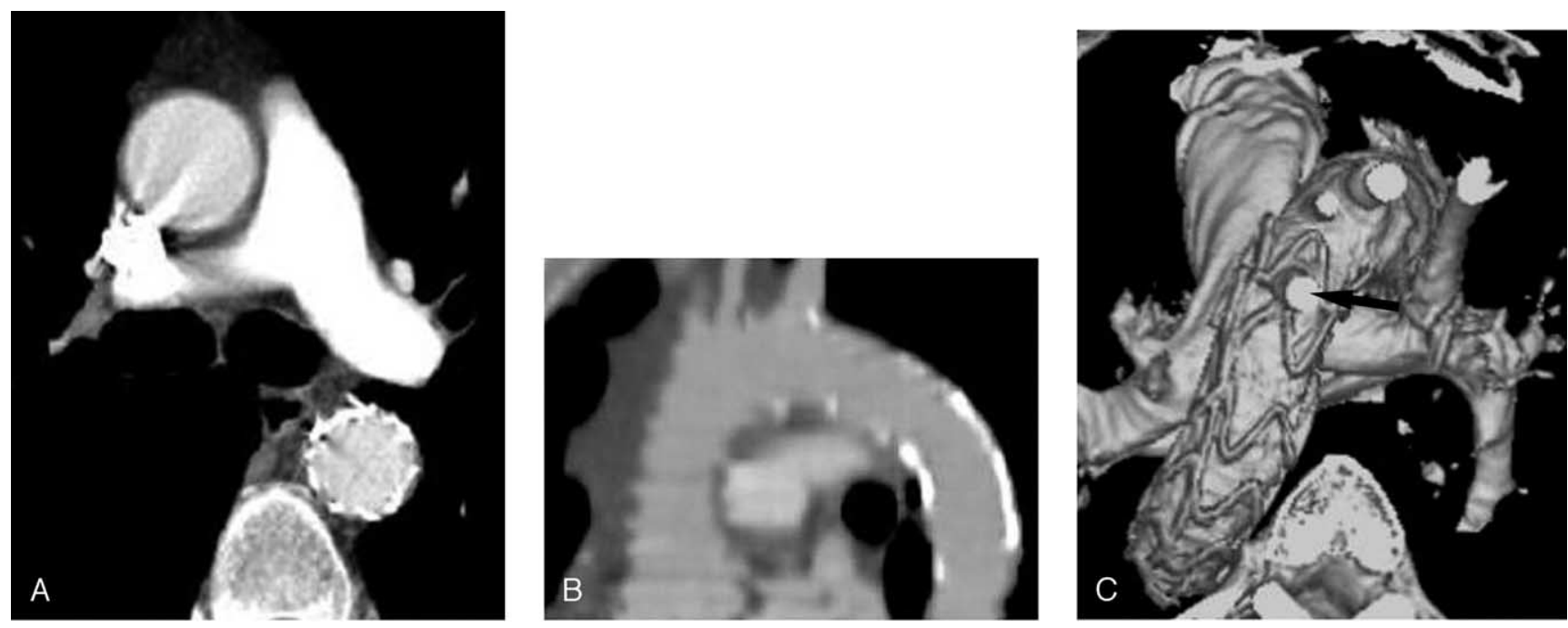

Figure 2. A, B, and C, Spiral CT with 3-dimensional shaded display reconstructions, 48 months after treatment, showing complete regression of the pseudoaneurysm and the initial noncovered portion of the stent graft over the left subclavian artery ostium, which stays patent. Arrow, Left subclavian artery ostium.

In group $1 b, 7$ patients were treated initially with intensive modalities, and the aortic lesion was monitored. Immediate continuous monitoring of arterial blood pressure and of vital signs and electrocardiogram was performed. Conservative treatment consisted primarily of the administration of $\beta$-blockers. All major associated injuries were treated surgically before repairing the aorta. The evolution of the aortic ruptures was carefully monitored in all patients with serial chest radiographs and angio-computed tomography (CT) scans. Aortic operation was scheduled after the resolution of all other significant associated injuries. In all patients, the aorta was replaced by prosthesis between 5 days and 257 days after the trauma (average 66 days). In 6 patients, extracorporeal circulation was used. One patient underwent surgery with a simple clamping technique.

\section{Stent Grafts Group 2}

In group 2, 29 patients (mean age: $37 \pm 19$ years) were treated with stent grafting. The use of stent grafts for this indication was approved by our ethical committee, and informed consent was obtained in all cases, either from the patients themselves or their relatives.

Stent grafting was classified in 8 cases as acute $(<14$ days $)$ according to Kato et al's classification of traumatic thoracic aortic injuries. ${ }^{10}$ The mean postinjury time period before stent grafting was $70 \pm 72$ days (1-245 days), with the procedure being performed within 24 hours in 2 patients.

Diagnosis and feasibility of stent grafting were assessed by spiral contrast-enhanced CT in all patients. The endovascular option was considered only for patients with a contained rupture and a proximal neck with a length of normal appearance of greater than $5 \mathrm{~mm}$.

Stent grafting was performed after surgical treatment of nonaortic, life-threatening lesions in 7 patients; 22 patients underwent stent grafting as primary treatment.
All patients were treated with the implantation of 1 unique self-expanding stent graft: the Excluder (W. L. Gore \& Associates, Inc., Flagstaff, Ariz) $(n=4)$, the Talent (Medtronic, Inc, Minneapolis, Minn) $(\mathrm{n}=23)$, or the Vanguard device (Boston Scientific Corporation, Natick, Mass) $(n=2)$. Stent grafts were oversized by $15 \%$ to achieve a tight friction seal, and the length was $2 \mathrm{~cm}$ longer than the lesion treated. The endovascular devices used were straight stent grafts of 24 to $36 \mathrm{~mm}$ in diameter and 80 to $100 \mathrm{~mm}$ in length. The introduction caliber of the compressed stent grafts was $18 \mathrm{~F}$ to $24 \mathrm{~F}$ (ie, $6-7.2 \mathrm{~mm}$ in diameter).

A team of interventional radiologists and cardiovascular surgeons performed the endovascular procedure with general anesthesia, tracheal intubation, and mechanical ventilation. The procedure was conducted in a sterile radiologic vascular interventional suite in accordance with the World Health Organization's recommendations on air handling in surgical suites.

The technique has been described. ${ }^{11}$ Briefly, a femoral artery was surgically isolated, and a transverse arteriotomy was performed after an intravenous bolus of 5000 IU heparin. Periprocedural transesophageal echocardiography (TEE) was performed to guide the stent-grafting procedure before and during deployment of the device. An initial aortogram (Advantx LCA, GE Medical Systems, Waukesha, Wis) with a $5 \mathrm{~F}$ pigtail catheter introduced through the brachial access helped to visualize the arterial anatomy. A 260-cm-long, 0.035-inch stiff guidewire (Backup, Meditech, Anaheim, Calif) was advanced up to the aortic arch under fluoroscopic guidance. The delivery system was positioned at the preestablished level in front of the aortic tear. A mean arterial pressure less than $70 \mathrm{~mm} \mathrm{Hg}$ was maintained during implantation, and the outer sheath was slowly withdrawn to fully deploy the implant. Thereafter, a compliant balloon included with the stentgraft package was inflated to fully anchor the stent into the nonaneurysmal wall of the aorta. 

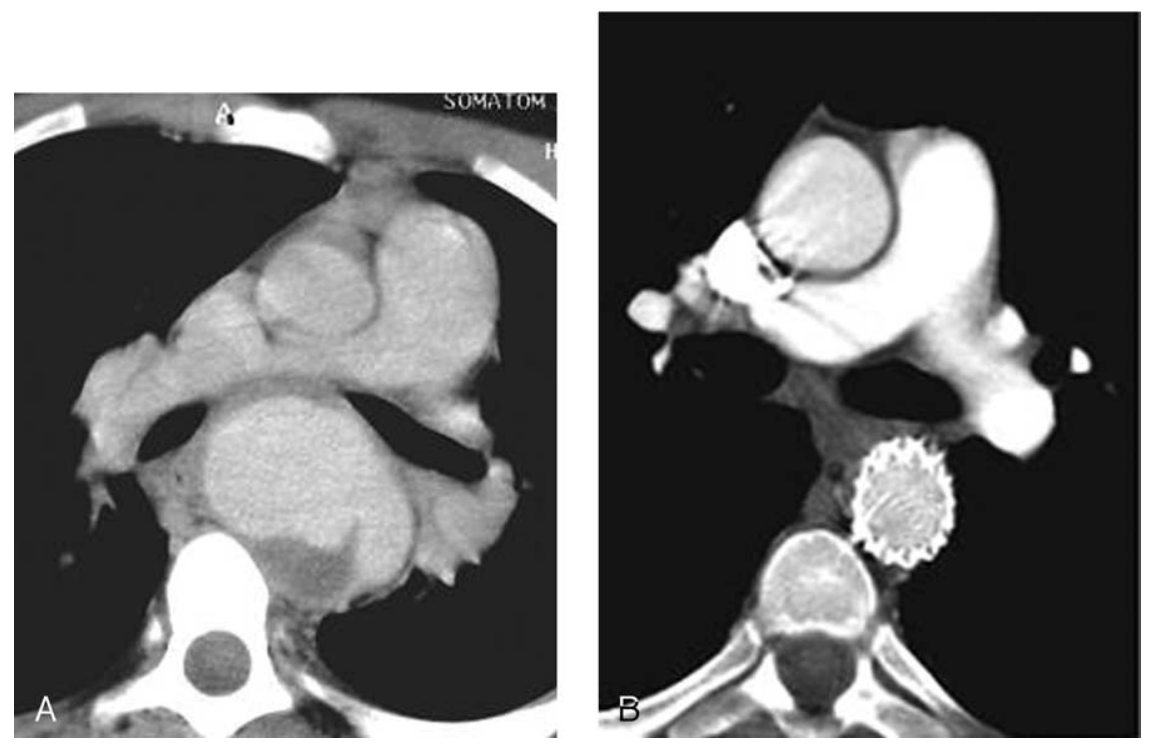

Figure 3. A, Thoracic spiral CT, 5 months after trauma, showing a well-delineated and partially thrombosed pseudoaneurysm of the proximal descending aorta measuring $4 \times 5 \mathrm{~cm}$. The true aortic lumen, left main stem bronchus, and right pulmonary artery are compressed by the pseudoaneurysm. B, Thoracic spiral CT, 84 months after treatment, showing complete regression of the pseudoaneurysm and good apposition of the stent graft to the aortic wall and open left bronchus.

In 6 patients, the proximal part of the stent graft was placed below the ostium of the left subclavian artery. In 17 patients, the stent graft was deployed with the first proximal noncovered end on the left subclavian artery ostium because of the close proximity of the pseudoaneurysmal sac. As long as only the bare portion of the stent graft covered the ostium of the left subclavian artery, this artery remained patent in all cases. The origin of the left subclavian artery was intentionally overstented in 6 patients without significant clinical consequence, and therefore a carotid to subclavian artery bypass was not needed.

Finally, the introducer delivery system was removed, and the arteriotomy was repaired after arteriographic and TEE controls (Figure 1,C). Anticoagulation was maintained for 48 hours and then followed by aspirin $250 \mathrm{mg}$ per day.

Further imaging follow-up consisted of TEE and spiral CT (before discharge; at 3, 6, and 12 months; and annually) after the intervention (Figures 2 and 3). Mean follow-up was 46 months (13-90 months).

\section{Conservative Group 3}

In group 3, 6 patients with minor, stable aortic lesions (defined as a small $[<1 \mathrm{~cm}]$ intimal flap with no or minimal periaortic hematoma) were treated medically and closely observed with clinical and radiologic follow-up (magnetic resonance imaging).

\section{Statistical Analysis}

Data were reexpressed as mean \pm SE. One and 2-way analyses of variance were performed to test the interaction between treatments and/or ISS and complications. Differences between treatments were determined with the unpaired $t$ test. All statistical analyses were performed with StatView (SAS Institute Inc, Cary, NC) and Super ANOVA softwares (ABACUS Concepts Inc, Berkeley, Calif).

\section{Results}

\section{Surgical Group 1}

In the group of patients who underwent emergency surgery, there were 4 intraoperative and 2 postoperative deaths $(21 \%)$. The cause of the intraoperative mortality was uncontrollable hemorrhage in 2 patients and irreversible cardiac arrest in 2 patients. One patient died of severe pulmonary insufficiency on day 2, and 1 patient died of sepsis on day 9. Furthermore, significant complications occurred only in this subgroup $(11 \%)$. These were 2 cases of paraplegia and 1 case of paraparesis. No difference in term of major complications was observed between the 2 surgical techniques.

In the delayed surgical repair group, there were only minor complications and no deaths.

\section{Stent Grafts Group 2}

Digital subtraction angiography and perioperative TEE revealed complete exclusion of the pseudoaneurysm in all patients, except for 1 patient with an immediate, minor, proximal endoleak. This endoleak spontaneously resolved on CT follow-up after 1 month. Only 1 stent graft was required in each patient. The mean procedure time was $96 \pm$ 24 minutes (median 83 minutes, range 75-180 minutes). 
No significant kinking, twisting, stenosis, intragraft thrombosis, migration, perigraft leak, pseudoaneurysmal expansion, or rupture was observed. In 1 case an iliac bypass was performed during the same procedure for an iliac rupture. No death, neurologic complication, or infection was observed, but early after intervention, fever, neutrophilic hyperleukocytosis, and biological inflammatory syndrome for 1 to 5 days (mean, 2.7 days) were observed in 6 patients; no causal infectious agent could be identified. Blind broad-spectrum antibiotics were given to the first 2 patients to cover an eventual stent-graft infection. One major complication of acute compression of the left main bronchus and homolateral pulmonary atelectasis occurred soon after the procedure; it was believed to be related to a sudden increase in pressure inside the freshly thrombosed pseudoaneurysmal sac. An endobronchial silicone stent was placed with good clinical and bronchoscopic results; it was retrieved 3 months later. With a 7-year follow-up, this patient presents no sequelae.

During the mean follow-up of 46 months, no intervention-related morbidity or mortality occurred. Correct positioning of stent graft and complete sealing of aortic rupture were found in all cases on follow-up TEE and CT control examinations (Figures 2 and 3 ).

\section{Conservative Group 3}

Six patients with minor aortic lesions never underwent aortic repair. No patient experienced alteration of the mediastinal silhouette during the medical treatment regimen, and no patient died of sudden exsanguination as a result of rupture of the traumatic pseudoaneurysm of the thoracic aorta.

\section{Discussion}

Rupture of the thoracic aorta as the result of blunt chest trauma leads to immediate death in $75 \%$ to $90 \%$ of cases $^{2,13}$ and accounts for up to $18 \%$ of deaths in motor vehicle accidents. ${ }^{14}$ Approximately $15 \%$ to $20 \%$ of the victims reach the hospital alive. ${ }^{15}$ For many decades, standard surgical practice has dictated that traumatic rupture of the thoracic aorta must be diagnosed quickly and managed aggressively with immediate surgical repair.

Despite advances in surgical and reanimation techniques, surgery for acute aortic rupture is still associated with significant morbidity and a high mortality rate up to $50 \%{ }^{1,16}$ Moreover, the systemic anticoagulation required for the extracorporeal circulation, even at a reduced dose as with heparin-coated systems, is undesirable in patients who have experienced trauma and have multiple fractures and/or parenchymatous or intracerebral lesions.

Moreover, controversy remains regarding the best method of management. ${ }^{5-9,17-23}$ In a review of the American literature, Walker and Pate ${ }^{24}$ found 64 patients with thoracic aortic trauma who were treated initially with conservative therapy; free rupture of the periaortic hematoma did not occur in any of the patients. In fact, in the majority of patients who reach the hospital alive, the adventitia and surrounding mediastinal structures remain more or less intact, thus partially preserving the integrity of the disrupted aorta and preventing acute exsanguination into the thorax. These and other data imply that the risk of rupture is not excessively high if the patient is clinically stable, especially if the tear is not circumferential.

Although urgent operative repair of confirmed blunt aortic injuries is recommended, delayed repair may be considered in select patients who may not necessarily benefit from immediate repair, including patients with severe head injury, infections, and severe multisystem trauma with poor physiologic reserve. Repair of the aortic injury may be undertaken once the patient has achieved a physiologic stabilization. On the basis of these data, a number of centers, including ours, have reconsidered therapeutic strategies, emphasizing the need to bring the patient to surgery in optimal clinical condition after the management of the associated lesions. ${ }^{5-9,18-25}$ In this study, no patient who was managed conservatively experienced alteration of the mediastinal silhouette during the medical treatment regimen, and no patient died of sudden exsanguination as a result of rupture of the traumatic pseudoaneurysm of the thoracic aorta. We believe that a conservative approach could be considered for limited, stable pseudoaneurysms, but close follow-up and avoidance of hypertension with the use of $\beta$-blockade are recommended. With this conservative strategy, no deaths were reported, either in the hospital or at home.

Currently, less-invasive strategies are being investigated for elective therapy of thoracic aortic lesions with the use of endovascular stent grafts. The theoretic advantages of endovascular treatment of acute rupture of the thoracic aorta are multiple. A simple arteriotomy is performed without thoracotomy or aortic clamping. The risk of medullar ischemia is theoretically less than that of conventional surgical intervention, ${ }^{10}$ possibly because of the absence of aortic clamping and the location of the aortic injury. Because up to $85 \%$ of traumatic aortic transections affect the segment of aortic isthmus, ${ }^{26}$ only a few branches to the spinal cord might be covered by implanted stent graft. Furthermore, severe head injury and pulmonary contusions pose fewer problems with this approach because, for the former, the absence of aortic crossclamping prevents an intracranial pressure increase, and for the latter, there is no need for intraoperative 1-lung ventilation. And finally, because stent-graft insertion can be performed with mild anticoagulation, the major bleeding complications observed with heparinization can theoretically be avoided.

These results confirm the results of other limited series. ${ }^{10,11,27-36}$ Every pseudoaneurysm was treated in 1 step with only 1 stent graft, and no leak was observed. There was no major coexisting morbidity, including such complications as heart failure, renal failure, distal embolization, or infection. None of the patients exhibited paraplegia or associated neurologic complications. This comparative study 
(with similar lesions and ISS scores) seems to confirm that stent grafting for traumatic aortic rupture is an advantageous alternative to conventional open surgery. Finally, because we can have the stent grafts in stock, the aortic repair could be performed in an emergency. Of course, the major limitation of these studies is the fact that they are not randomized studies, but such a study seems difficult to undertake.

In conclusion, of course a patient with signs of imminent rupture is considered for immediate treatment, but this study supports previous reports that free rupture of a contained acute traumatic rupture of the thoracic aorta from blunt trauma is unlikely to occur under proper monitoring and control of blood pressure. Also, this study suggests that patients who are treated in this delayed manner have better outcomes, with fewer intraoperative and postoperative complications. Finally, this comparative study seems to confirm that stent grafting of the aortic isthmus is a valuable therapeutic alternative to surgical repair, especially in patients considered high risk for conventional thoracotomy. Longterm follow-up is necessary to determine the role of stent grafts in preventing late aortic rupture.

\section{References}

1. Fabian TC, Richardson JD, Croce MA, et al. Prospective study of blunt injury: multicenter trial of the American Association for Surgery of Trauma. J Trauma. 1997;42:374-80.

2. Parmley LF, Mattingly TW, Marian WC. Non-penetrating traumatic injury of the aorta. Circulation. 1958;17:1086-100.

3. Avery JE, Hall DP, Adams JE, et al. Traumatic rupture of the aorta. South Med J. 1979;72:1240-5.

4. Kouchoukos NT, Dougenis D. Surgery of the thoracic aorta. $N$ Engl J Med. 1997;336:1876-88.

5. Akins CW, Buckley MJ, Dagget W. Acute traumatic disruption of the thoracic aorta: a ten year experience. Ann Thorac Surg. 1981;31:305-9.

6. Kipfer B, Leupi F, Schuepbach P, et al. Traumatic rupture of the thoracic aorta: immediate or delayed surgical repair? Eur J Cardiothorac Surg. 1994;8:30-3.

7. Pate JW, Fabian TC, Walker W. Traumatic rupture of the aortic isthmus: an emergency? World J Surg. 1995;19:119-26.

8. Maggisano R, Nathens A, Alexandrova N. Traumatic rupture of the thoracic aorta: should one always operate immediately? Ann Vasc Surg. 1995;9:44-52.

9. Fisher RG, Oria RA, Mattox, KL, et al. Conservative management of aortic lacerations due to blunt trauma. J Trauma. 1990;30:1562-6.

10. Kato N, Dake MD, Miller DC, et al. Traumatic thoracic aortic aneurysm: treatment with endovascular stent-grafts. Radiology. 1997;205: 657-62.

11. Rousseau H, Soula P, Perreault P, et al. Delayed treatment of traumatic rupture of the thoracic aorta with endoluminal covered stent. Circulation. 1999;99:498-504.

12. Baker SP, O'Neill B, Haddon W Jr, et al. The injury severity score: a method for describing patients with multiple injuries and evaluating emergency care. J Trauma. 1974;14:187-96.

13. Williams JS, Graff JA, Uku JM, et al. Aortic injury in vehicular trauma. Ann Thorac Surg. 1994;57:726-30.
14. Greendyke RM. Traumatic rupture of aorta. Special reference to automobile accidents. JAMA. 1996;195:119-22.

15. Merrill WA, Lee RB, Hamman JW, et al. Surgical treatment of acute traumatic tear of the thoracic aorta. Ann Surg. 1988;207:699-706.

16. Von Oppell UO, Dunne TT, DeGroot MK, et al. Traumatic aortic rupture: 20-year meta-analysis of mortality and risk of paraplegia. Ann Thorac Surg. 1994;58:585-93.

17. Butler KL, Moore EE, Harken AH. Traumatic rupture of the descending aorta. AORN J. 1996;63:917-25.

18. Prêtre R, Chilcott M. Blunt trauma to the heart and great vessels. N Engl J Med. 1997;336:626-32.

19. Stulz P, Reymond MA, Bertschmann W, et al. Decision-making aspects in the timing of surgical intervention in aortic rupture. Eur J Cardiothorac Surg. 1991:5:623-7.

20. Striffeler H, Leupi F, Kaiser G, et al. Traumatic rupture of the thoracic aorta in childhood with special reference to the therapeutic strategy. Eur J Pediatr Surg. 1993;3:50-3.

21. Galli R, Pacini D, Di Bartlomeo R, et al. Surgical indications and timing of repair ruptures of the thoracic aorta. Ann Thorac Surg. 1998;65:461-4.

22. Frykberg ER, Crump JM, Dennis JW, et al. Nonoperative observation of clinically occult arterial injuries: a prospective evaluation. Surgery. 1991;109:85-96.

23. Soots G, Warembourg H Jr, Prat A, et al. Acute traumatic rupture of the aorta: place of delayed surgical repair. J Cardiovasc Surg. 1989: 30:173-7.

24. Walker WA, Pate JW. Medical management of acute traumatic rupture of the thoracic aorta. Ann Thorac Surg. 1990;50:965-7.

25. Blegvad S, Lippert $\mathrm{H}$, Lund $\mathrm{O}$, et al. Acute or delayed surgical treatment of traumatic rupture of the descending aorta. J Cardiovasc Surg. 1989:30: 559-64.

26. Duhaylongsod FG, Glower DD, Wolfe WG. Acute traumatic aortic aneurysm: the Duke experience from 1970 to 1990. J Vasc Surg. 1992;15:331-43.

27. Perreault $\mathrm{P}$, Soula $\mathrm{P}$, Rousseau $\mathrm{H}$, et al. Acute traumatic rupture of the thoracic aorta: delayed treatment with endoluminal covered stent. A report of two cases. J Vasc Surg. 1998;27:538-44.

28. Deshpande A, Mossop P, Gurry J, et al. Treatment of traumatic false aneurysm of the thoracic aorta with endoluminal grafts. $J$ Endovasc Surg. 1998;5:120-5.

29. Schonholz C, Donnini F, Naselli G, et al. Acute rupture of an aortic false aneurysm treated with a stent-graft. J Endovasc Surg. 1999;6: 293-6.

30. Ahn SH, Cutry A, Murphy TP, et al. Traumatic thoracic aortic rupture: treatment with endovascular graft in the acute setting. J Trauma. 2001;50:949-51.

31. Fujikawa T, Yukioka T, Ishimaru S, et al. Endovascular stent grafting for the treatment of blunt thoracic aortic injury. I Trauma. 2001;50: 223-9.

32. Hoffer EK, Karmy-Jones R, Bloch RD, et al. Treatment of acute thoracic aortic injury with commercially available abdominal aortic stent-grafts. J Vasc Interv Radiol. 2002;13:1037-41.

33. Doss M, Martens S, Hemmer W. Emergency endovascular interventions for ruptured thoracic and abdominal aortic aneurysms. Am Heart J. 2002;144:544-8.

34. Fattori R, Napoli G, Lovato L, et al. Indications for, timing of, and results of catheter-based treatment of traumatic injury to the aorta. Am J Roentgenol. 2002;179:603-9.

35. Lachat M, Pfammatter T, Witzke H, et al. Acute traumatic aortic rupture: early stent-graft repair. Eur J Cardiothorac Surg. 2002;26: 959-63.

36. Thompson CS, Rodriguez JA, Ramaiah VG, et al. Acute traumatic rupture of the thoracic aorta treated with endoluminal stent grafts. J Trauma. 2002;52:1173-7. 